\title{
Die „Großfürsten der Libu“ im westlichen Delta in der späten 22. Dynastie
}

\author{
KarlJansen-Winkeln \\ Ägyptologisches Seminar Freie Universität Berlin \\ jawinkel@fu-berlin.de
}

\begin{abstract}
In a recent article on the "Great chiefs of the Libu" in the Western Delta, Jan Moje tried to show that there were two local rulers called Ni-ma-teped (A/B) and two Tjer-pa-hati (I/II), attested on five donation stelae. If one of those stelae is ascribed to Shoshenq IV rather than to Shoshenq $\mathrm{v}$, three stelae mention a (single) ruler Ni-ma-teped during the reign of Shoshenq IV, and the remaining two a ruler Tjer-pa-hati under Shoshenq v.
\end{abstract}

\section{Keywords}

Shoshenq IV - Shoshenq v - Great Chief - Libu - Donation stelae - 22nd Dynasty Western Delta

1. AlsJEAn YoYotTe seine grundlegende Arbeit über die libyschen Fürstentümer im Delta ${ }^{1}$ verfasste, schien ein wr $3 n$ Rbw namens $N$ 3ym 3 tjpd (o.ä.) zeitlich aus dem Rahmen zu fallen: die Schenkungsstele St. Petersburg $5630,{ }^{2}$ auf der er als

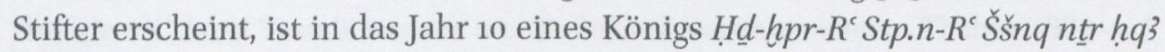
$J w n w$ datiert, was nach damaligen Stand eigentlich Schoschenk I. sein musste. ${ }^{3}$ Allerdings war schon ҮочОтте wegen des sonst bei Schoschenk I. nicht üblichen Zusatzes ntr hqq3 Jwnw (und vermutlich auch wegen des ungewöhnlich frühen Datums, weit mehr als 100 Jahre vor dem nächsten Beleg für einen

1 Yoyotte, "Les principautés ${ }^{4}$.

2 Jansen-Winkeln, Inschriften, 11,257 (26.6).

3 Daher auch von Gauthier, Livre des rois, III, 308 (v) unter Schoschenk I. eingeordnet. 
„Großfürst der Libyer") abgeneigt, diese Stele unter Schoschenk I. zu datieren: Er vermutete, sie sei tatsächlich Schoschenk III. zuzuweisen, bei dem sich der Zusatz $n t r$ h $h q^{3} J w n w$ häufig findet, und der Thronname $H \underline{d} d-h p r-R^{c} S t p \cdot n-R^{c}$ sei gedankenlos von Takeloth II, dem (angeblichen) Vorgänger Schoschenks III. übernommen worden. ${ }^{4}$

Diese Ansicht hat sich allerdings nicht durchgesetzt: Kenneth Kitchen hat überzeugende Gründe dagegen vorgebracht, ${ }^{5}$ dementsprechend findet sich der Großfürst „Niu-mateped $\mathrm{A}^{4}$ von dieser Stele in seiner "table $21^{\text {“6 }}$ im respektablen Abstand von 136-146 Jahren zum zeitlich nächst bezeugten wr 3 Rbw namens Jnj-Jmn-n3j.f-nbw (A) aus dem Jahr ${ }_{31}$ Schoschenks III. eingeordnet.

Auf einer von Wilhelm SPIEgelberg publizierten Schenkungsstele aus Kôm Firin ${ }^{7}$ kommt ein wr ' $n$ Rbw vor, dessen ungewöhnlicher Name m3tppd eigentlich derselbe sein sollte wie auf der Stele St. Petersburg 5630, und er wird

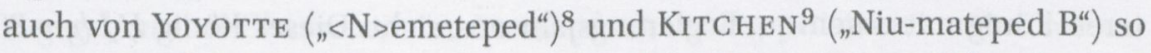
verstanden. ${ }^{10}$ Diese Stele stammt aus dem Jahr 8 eines Königs Schoschenk $z 3$ B3stt Mrij-Jmn ntr h hq3 W3st, der von YoYоTTE mit gewissem Vorbehalt („probablement") mit Schoschenk v. identifiziert worden war, da Schoschenk v. zuweilen diese Zusätze führt." Diese Gleichsetzung ist bis heute aufrechterhalten worden.

Ein dritter Beleg für diesen Namen, dieses Mal Nm3rtpd geschrieben, findet sich auf der 1974 von ERHART GRAEFE veröffentlichten Schenkungsstele Hildesheim RP 5382 (damals in einer Kölner Privatsammlung) ${ }^{12}$ aus dem Jahr 4 eines Königs Mrij-Jmn z3 B3stt Ššnq. Auch diese Stele dürfte aus dem westlichen Delta stammen. Auf ihr sind allerdings die Titel dieses Mannes nicht erhalten. Was die Identität des Königs angeht, so hielt GRAEFE Schoschenk III-V. ${ }^{13}$ für

\footnotetext{
4 Yoyotte, „Les principautés“, 142-43( $\$ 29)$.

5 Kitchen, The Third Intermediate Period, § 249, n. 278, ebenso mit ausführlichen Belegen Bonhême, Les noms royaux, 104-05.

6 Kitchen, The Third Intermediate Period, 490.

7 Spiegelberg, „Neue Schenkungsstelen“, $57-58$, Taf. V.

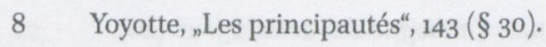

9 Kitchen, The Third Intermediate Period, § 311.

10 Colin, Les Libyens en Égypte, II, 55-56, gibt Nmrtpd als eine Art "Normalform“ dieses Namens, ebenso Winnicki, Late Egypt and Her Neighbours, 403-04, der ihn als „Nmrt, der Libyer" deutet.

11 Yoyotte, „Les principautés“, § 30, mit n. 7 .

12 Graefe, „Eine neue Schenkungsstele“.

13 Mit Schoschenk IV. war damals der oberägyptische Schoschenk Wsr-m3't-R' gemeint, der heute als der vi. numeriert wird.
} 
möglich und nur Schoschenk I. für ausgeschlossen, während KITCHEN ${ }^{14}$ diesen „Niuma(r)tiped C“ am ehesten unter Schoschenk III. datieren wollte.

2. Das Problem des großen zeitlichen Abstands zwischen dem angeblich ersten Beleg schon unter Schoschenk I. und dem zweiten aus dem Jahr 31. Schoschenks III. wurde 1993 von AIDAN DoDson gelöst, ${ }^{15}$ der seinerseits auf Vorschläge von DAvid RoHL zurückgriff. Demnach hat zwischen Schoschenk III. (Wsr$m 3^{3} t-R^{c}$, öfter mit Zusatz $z^{3}$ B3stt und $h q^{3} J w n w$ in der zweiten Kartusche) und Pami (nachweislich der Vater seines Nachfolgers Schoschenks v.) ${ }^{16}$ ein weiterer König namens Schoschenk mit dem Thronnamen $H \underline{d}-h p r-R^{c}$ regiert. Da König Pami 25 Jahre nach dem 28. Regierungsjahr Schoschenks III. den Thron bestieg ${ }^{17}$ und für Schoschenk III. das Jahr 39 sein letztes sicher bezeugtes ist. ${ }^{18}$ bleibt zwischen Schoschenk III. und Pami ein Zeitraum, der für einen weiteren König mit einem 10. Regierungsjahr ausreicht. Dieser König $H \underline{d}$ - $h p r-R^{c}$ Schoschenk (nach neuer Zählung IV.) ist nun allgemein anerkannt. ${ }^{19}$

Also könnte der Fürst der Libyer Nmrtpd in den Jahren 4 (Stele Hildesheim) und 10 (Stele St. Petersburg) Schoschenks Iv. belegt sein sowie zuletzt im Jahr 8 Schoschenks v. Das ergäbe eine "Regierungszeit" von mindestens 24-26 Jahren, kein unrealistischer Zeitraum.

3. Auf einer Schenkungsstele (heute Brooklyn 67.119) aus dem Jahr 15 Schoschenks v. ${ }^{20}$ ist dann schon ein neuer "Großfürst der Libyer" belegt, den YoyotTe und Kitchen „Te(?)ter, fils de Ded(?)“ bzw. „Titaru son of Didi“ gelesen hatten. Eine 1991 im Handel angebotene und schließlich vom вм London erworbene weitere hieratische Schenkungsstele (EA 73965) ${ }^{21}$ erwähnt als Stifter offenbar denselben Mann in einer nur leicht variierenden Schreibung, und zwar hieroglyphisch im Bildfeld (5\%) und hieratisch im Haupttext. Ein Vergleich der Schreibungen zeigt deutlich, dass sich die bisherige Lesung Ttr $p$ ?

14 Kitchen, The Third Intermediate Period, $\S 517$.

15 Dodson, "A New King Shoshenq Confirmed?"

16 Serapeumstele Louvre IM 3049, s. Malinine, u.a., Catalogue des stèles, 25-26 (26).

17 Malinine, u.a., Catalogue des stèles, 21-25, Stelen Nr. 22-25; Kitchen, The Third Intermediate Period, $\$ 82$.

18 Jansen-Winkeln, Inschriften, $I I, 183$ (22.12), 203 (22.38).

19 Vgl. Kitchen, The Third Intermediate Period, xxv-XxvI (§ Y); Kitchen, „Third Intermediate Period: Fact \& Fiction“, 166, 197, 202.

20 Yoyotte, „Les principautés“, § 32, pl.I (2); vollständig veröffentlicht von Kitchen, „Two Donation Stelae", 64-67, Fig.4.

21 Quirke und Spencer, British Museum Book, 201; Spencer, Kom Firin I, Taf. 89; hieroglyphischer Text in Jansen-Winkeln, Inschriften, II, 274 (28.17). 
$D\left(j d(j)\right.$ nicht halten läßt, sondern dass es sich um einen Namen $T(n) r-p 3-h h^{3} w t j$ (o.ä.) handelt. ${ }^{22}$ Allerdings sind die genaue Umschrift und das Verständnis dieses Namens problematisch, da vor allem die beiden hieratischen Schreibungen etwas entstellt sind, wie das im Steinhieratischen der Schenkungsstelen ja nicht selten vorkommt. Beim hieroglyphischen Beleg ist nur das letzte Zeichen unter dem $h 3 t$ unsicher: JAN MOJE möchte darin das Stadtdeterminativ (O 49) erkennen, ${ }^{23}$ aber je nach Beleuchtung könnte man es auch " lesen. ${ }^{24}$ Der hie-

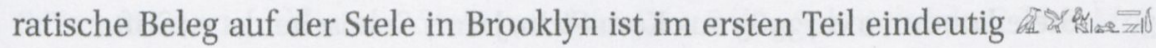

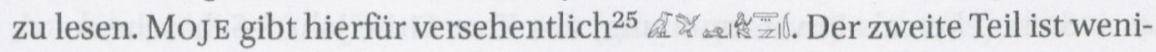
ger klar. Das nächste Zeichen oben ist trotz der recht ungewöhnlichen Formder linke Teil fast rund und etwas abgetrennt-sicher das ḩ3t-Zeichen (F 4). ${ }^{26}$ Darunter lese ich zunächst, mit KiTcHEN, ein etwas beschädigtes 9 , kein t. Die drei unförmigen Kreise daneben kann vielleicht man als $\|_{\circ}$ deuten, aber das ist zugegeben eher Glaubenssache. Von der letzten Gruppe ist nur ein Strich deutlich, den Kreis darüber liest MoJE als Stadtdeterminativ ( $\mathrm{O} 49$ ), ich halte es

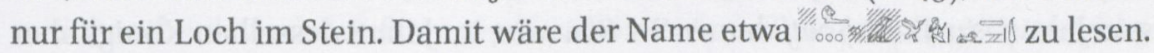

Auf der Londoner Stele (Z.4) ist wiederum der Anfang des Namens recht deutlich geschrieben ( lich. Das letzte Zeichen oben sieht mit MoJe in der Tat wie das hieroglyphische Stadtdeterminativ $\left(\mathrm{O}_{49}\right)$ aus und das Zeichen davor wie eine Hälfte der Himmelshieroglyphe ( $\mathrm{N}_{1}$ ), aber das ergibt keinen Sinn. ${ }^{27}$ Ich halte es nach wie vor für besser, die beiden letzten Zeichen oben auch hier für ein (sehr) missglückte Wiedergabe des ḩ3t-Zeichens (das ja auch auf der Brooklyner Stele etwas zerteilt und in der linken Hälfte rund ist) anzusehen und $h h^{3} w t j($ ( lesen. Ich würde daher diesen Namen als tr bzw. tnr-p $3-h \zeta^{3} w t j$ „Der Anführer ist stark“ (oder: „möge stark sein“) verstehen. ${ }^{28}$

4. Wie auch immer man diesen Namen umschreibt und versteht, es gibt (bis jetzt) folgende Schenkungsstelen, auf denen libysche Großfürsten mit den

22 S. Jansen-Winkeln, Inschriften, II, 274, 502, unten.

23 Moje, „British Museum EA $73965 “, 85,87$.

24 Eigentlich in der falschen Richtung, was aber bei nach links ausgerichteten hieroglyphischen Texten recht häufig ist.

25 Mündliche Mitteilung J. Moje.

26 So auch Moje, „British Museum EA $73965 “, 87$.

27 Vgl. auch Moje, „British Museum EA 73965“, 88.

28 Vgl. Ranke, Personennamen, 381, 19 und 21-22. Da ja zumindest der Artikel $p 3$ eindeutig ägyptisch ist, kann man ausschließen, dass der Name libysche Morpheme enthält, da es m.W. keine hybriden libysch-ägyptischen Namen gibt. 
Namen Nmrt-pd und Tr-p3-h3tj verzeichnet sind, in der Reihenfolge der bisher akzeptierten Daten:

1. Stele Hildesheim 5382 (wohl aus dem Westdelta), Jahr 4 Schoschenks IV., Stifter $N$.

2. Stele St. Petersburg 5630 (wohl aus dem Westdelta), Jahr 10 Schoschenks IV.; Stifter $N$.

3. Stele London вм 73965 aus Kom Firin, Jahr 7 Schoschenks V.; Stifter: $T$.

4. Stele (ehemals Privatsammlung) aus Kom Firin, Jahr 8 Schoschenks v.; Stifter $N$.

5. Stele Brooklyn 67.119 aus Kom Firin, Jahr 15 Schoschenks v.; Stifter $T$.

Durch die Datierung der Londoner Stele in das Jahr 7 Schoschenks v. ergibt sich nun eine Schwierigkeit für die bisherige Reihung der "Großfürsten der Libyer", die zuerst MoJE erkannt und zu lösen versucht hat: ${ }^{29}$ Wenn in diesem Jahr 7 ein $T r-p 3-h 3 w t j$ der wr ' $n$ Rbw war, im Jahr 8 desselben Königs aber (N3y)m3tjpd und im Jahr 15 wiederum (ein) Tr-p3-ḩ3wtj, sollte es sich bei dem Vorgänger und dem Nachfolger des N3ym3tjpd um zwei verschiedene Personen handeln. Da aber ein N3ym3tjpd (bzw. Nmrt-pd) auch in den Jahren 4 und 10 Schoschenks Iv. bezeugt ist, muss es sich in der Zeit von Schoschenk Iv. bis zum Jahr 15. Schoschenks v. um eine Reihe von vier Lokalregenten handeln, in MoJes Wiedergabe: ${ }^{30}$

Ni-ma-teped A-Tjer-pa-hati I.-Ni-ma-teped B-Tjer-pa-hati II.

Das wäre nicht ausgeschlossen, zumal wenn es sich jeweils um Vater und Sohn handelte, denn die "Papponymie“, die Benennung des Sohnes nach dem eigenen Vater, war durchaus auch unter libyschen Fürsten üblich, z.B. bei den Lokalherrschern von Mendes ${ }^{31}$ und Busiris. ${ }^{32}$ Es ergäbe sich allerdings, und das ist ein Punkt, der Mojes These bedenklich erscheinen läßt, ein ziemliches Gedränge von „Fürsten der Libyer“ in dieser Zeit, denn im

\footnotetext{
29 Moje, „British Museum EA $73965^{4}$.

30 Moje, „British Museum EA 73965 “, 91.

31 Harnacht A-Smendes IV - Harnacht B - Smendes v, s. Kitchen, The Third Intermediate Periode, 599 (Table 22A).

32 Shoshenq E-Pimay IV-Shoshenq F-Pimay V, s. Kitchen, The Third Intermediate Periode, 491 (Table 22D).
} 
Jahr 19 Schoschenks v. ist schon der nächste namens $\mathrm{Kr}$ belegt. ${ }^{33}$ Zwischen den Jahren 7 bis 19 Schoschenks v. wären nicht weniger als vier wr $3 n R b w$ unterzubringen, in zwölf Jahren also ein dreimaliger Wechsel. Obwohl das natürlich keineswegs ausgeschlossen ist, scheint diese Dichte doch nicht unbedingt wahrscheinlich zu sein.

5. Eine wesentlich einfachere Lösung ergibt sich, wenn man die von SPIEgelberg publizierte Stele aus dem Jahr 8 des Schoschenk $z 3$ B3stt MrijJmn nț hq 3 W3st (der Thronname wird nicht genannt) nicht Schoschenk V., sondern IV. zuordnet. Dies war schon einmal von DoDson erwogen worden, ${ }^{34}$ allerdings mit großer Zurückhaltung, weil bis dahin der Zusatz $h q 3$ W3st nur für Schoschenk v. belegt war ${ }^{35}$ und nicht für Schoschenk IV. Inzwischen gibt es aber einen Beleg aus Tanis ${ }^{36}$ für einen König $H \underline{d}$-hpr-R $R^{c}$ Stp.n-R $R^{c}$ Mrij-Jmn $z$ z B3stt Ššnq ntr hq ${ }^{3}$ W3st, unzweifelhaft Schoschenk IV. Die Belege für die beiden Zusätze $z 3$ B3stt und $h q 3$ W3st stellen sich nun folgendermaßen dar:

Schoschenk v. wird auf den "Sedfestblöcken“ aus Tanis oft als $z 3$ B3stt und $h q$; W3st bezeichnet, $h q 3$ W3st ist er auch auf einer Schenkungsstele aus Kom Firin ${ }^{37}$ und $z 3$ B3stt auf einer weiteren aus Kom Firin ${ }^{38}$ sowie auf Uschebtifragmenten aus dem Grab Osorkons II., ${ }^{39}$ sofern sie ihm tatsächlich zuzuweisen sind. ${ }^{40}$

Schoschenk IV. ist $z 3$ B3stt und $h q 3$ W3st ebenfalls auf einem Block aus Tanis, sicherlich nicht zufällig, da Tanis eben das nördliche Theben war. Das Beiwort $z 3$ B3stt führt er auch sonst öfter, in fünf Belegen (von acht insgesamt), ${ }^{41}$ während er außerhalb von Tanis offenbar nicht $h q^{3}$ W3st, sondern $h q^{3} J$ Jwnw genannt wird. ${ }^{42}$ Da nun Schoschenk v. in Tanis als $h q q^{3}$ W3st bezeichnet wird sowie auf einer Schenkungsstele aus Kom Firin, und Schoschenk IV. gleichfalls in Tanis $h q 3$ Wsst heißt, spricht nichts dagegen, die von SPIEgELBERG publizierte Schenkungsstele aus Kom Firin aus einem Jahr 8 Schoschenk IV. (statt v.)

33 Schenkungsstele Kairo JE 30972, s. Koenig, „Notes sur la stèle“; Jansen-Winkeln, Inschriften, II, 276-77 (28.21).

34 Dodson, „A New King Shoshenq Confirmed?“, 53 mit n. 8, 57 mit n. 36.

35 Auf den im Tempelsee von Tanis verbauten "Sedfestblöcken“, s. Montet, Le lac sacré de Tanis, pl. vII-XXVIII.

36 Jansen-Winkeln, Inschriften, $I I, 256$ (26.1).

37 Brooklyn 67.119, s. Kitchen, "Two Donation Stelae", 64-67, Fig.4; Jansen-Winkeln, Inschriften, $I I, 274$ (28.18).

38 London BM 73965, s. Jansen-Winkeln, Inschriften, II, 274 (28.17).

39 Jansen-Winkeln, Inschriften, II, 269 (28.2).

40 S. Dodson, "Some Notes", 224; Aston, Burial Assemblages of Dynasty 21-25, 58-59.

41 Jansen-Winkeln, Inschriften, II, 256-8 (26.1, 26.3-4, 26.7-8).

42 Jansen-Winkeln, Inschriften, II, 256-8 (26.3-6, 26.8). 
zuzuweisen: dann wäre er, wie Schoschenk v., jeweils auf einem Denkmal in Tanis und auf einer Schenkungsstele aus Kom Firin als $h q 3$ W3st bezeugt.

6. Unter dieser Voraussetzung ergäbe sich folgende Reihung der Stelen:

1. Stele aus Jahr 4 Schoschenks IV., Stifter $N$.

2. Stele aus Jahr 8 Schoschenks IV.; Stifter $N$.

3. Stele aus Jahr 10 Schoschenks IV.; Stifter $N$.

4. Stele aus Jahr 7 Schoschenks V.; Stifter $T$.

5. Stele aus Jahr 15 Schoschenks V.; Stifter $T$.

Das würde bedeuten, dass „Niumateped“ (Nmrt-pd) spätestens seit dem Jahr 4 Schoschenks IV. als libyscher Großfürst lokaler Regent war, möglicherweise schon etwas früher: Im 31. Jahr Schoschenks III. wird auf der Schenkungsstele Moskau I.1.a.5647 ${ }^{43}$ aus dem Delta ein P3-wrd, Sohn eines Jnj-Jmn-n3j.f-nbw, als $m s n$ wr $3 R b w$ bezeichnet, und im Bildfeld ist ein Jnj-Jmn-n3j.f-nbw anbetend vor den Göttern dargestellt. YоYОттЕ hatte daraus geschlossen, dass eben dieser Jnj-Jmn-n3j.f-nbw der wr $3 n$ Rbw sein muss, und dies ist seither offenbar allgemeine Ansicht. Dann wäre Nmrt-pd also irgendwann zwischen Jahr 31. Schoschenks III. und Jahr 4 Schoschenks IV. der Nachfolger des Jnj-Jmn-n3j.f$n b w$ geworden. ${ }^{44}$

MOJE meint allerdings, Jnj-Jmn-n3j.f-nbw sei zu diesem Zeitpunkt schon tot gewesen, da er selbst nicht mit dem Titel eines wr $\xi$ bezeichnet wird, sondern nur sein Sohn als Nachkomme eines Großfürsten. Dann müsste mit diesem Jahr 31 bzw. schon vorher sein Nachfolger, vermutlich Nmrt-pd, Lokalherrscher gewesen sein. Das ist möglich, aber keineswegs zwingend: In der Regel werden Würdenträger, auch wenn sie schon tot sind, noch mit den Titeln der Ämter bezeichnet, die sie zu Lebzeiten ausgeübt haben. Daher lässt sich umgekehrt kaum folgern, dass ein Funktionär oder Machthaber zu der Zeit nicht mehraktiv bzw. schon tot war, wenn nur sein Name (ohne Titel) erwähnt wird. Im übrigen wird auch der Großfürst $R w d-$-Jmn im Bildfeld einer Schenkungsstele ${ }^{45}$ nur mit seinem Namen (ohne Titel) benannt, auf einer anderen ${ }^{46}$ werden Großfürst

43 Hodjash und Berlev, The Egyptian Reliefs and Stelae, 157-58, 160, 164 (106); Jansen-Winkeln, Inschriften, II 202-03 (22.34).

44 Sofern es nicht noch einen kurzlebigen weiteren Großfürsten zwischen ihnen gab.

45 Berlandini, „Une stèle de donation“, Taf. 49-50; Jansen-Winkeln, Inschriften, II, 275-76 (28.20).

46 Kairo JE 30972, s. Koenig, „Notes sur la stèle“; Jansen-Winkeln, Inschriften, II, 276-77 (28.21). 
und Empfänger der Schenkung im Bildfeld nur mit ihren Titeln erwähnt, aber ohne ihre Namen. Offenbar war man mit den genauen Bezeichnungen auf den Schenkungsstelen nicht so penibel.

Wie auch immer, von Jahr 4 bis 10 Schoschenks IV. war Nmrt-pd der wr ' $\mathrm{n}$ $R b w$, und er könnte es auch schon einige Jahre vorher und noch einige nachher gewesen sein.

Der Großfürst Tr-p3-ḩiwtj ist seinerseits in den Jahren 7 und ${ }_{15}$ Schoschenks v. bezeugt, im Jahr 19 schon sein mutmaßlicher Nachfolger $\mathrm{Kr}$. Der Wechsel sollte also zwischen Jahr 15 und 19 erfolgt sein. Tr-p3-hiwtj könnte aber schon eine geraume Zeit vor dem Jahr 7 Großfürst gewesen sein, schon unter König Pami. Von der zeitlichen Erstreckung her ist es keineswegs nötig (wenn natürlich auch durchaus möglich), zwischen Nmrt-pd und Tr-p3-hiswtj einen weiterer Großfürsten der Libyer anzusetzen.

In jedem Fall ist es eine weit einfachere und näherliegende Lösung, die von SPIEgelberg publizierte Stele Schoschenk IV. zuzuweisen und damit von nur jeweils einem Großfürsten Nmrt-pd und Tr-p3-hiswtj auszugehen.

\section{Bibliographie}

Aston, D. Burial Assemblages of Dynasty 21-25. Wien: Verlag der Österreichischen Akademie der Wissenschaften, 2009.

Berlandini, J. „Une stèle de donation du dynaste libyen Roudamon“. BIFAO 78 (1978): 147-163, Taf.49-50.

Bonhême, M.-A. Les noms royaux de la Troisième Période Intermédiaire. BdE 98. Kairo: Institut français d'archéologie orientale du Caire, 1987.

Colin, F. Les Libyens en Égypte (XVe siècle A.C. $-I I^{e}$ siècle P.c.). Onomastique et histoire. Dissertation Université Libre de Bruxelles, 1996.

Dodson, A. „A New King Shoshenq Confirmed?“ GM 137 (1993): 53-58.

"Some Notes Concerning the Royal Tombs at Tanis". CdE 63 (1989): 221-233.

Gauthier, H. Le livre des rois d'Egypte. T.III: De la XIXe à la XXIV e dynastie. MIFAO 19. Kairo: Institut français d'archéologie orientale du Caire, 1914.

Graefe, E. „Eine neue Schenkungsstele aus der 22. Dynastie“. Armant. Deutsch-Arabische Kulturzeitschrift, Heft 12 (1974): 1-9.

Hodjash, S. und O. Berlev. The Egyptian Reliefs and Stelae in the Pushkin Museum of Fine Arts, Moscow. Leningrad: Aurora Art Publishers, 1982.

Jansen-Winkeln, K. Inschriften der Spätzeit. Teil II: Die 22.-24. Dynastie. Wiesbaden: Harrassowitz Verlag, 2007.

Kitchen, K.A. The Third Intermediate Periode in Egypte (noo-650 BC). Warminster: Aris \& Phillips LTD, 1996. 
- "The Third Intermediate Periode in Egypte: An Overview of Fact \& Fiction". In The Libyan Period in Egypt, Historical and Cultural Studies into the 21st-24th Dynasties: Proceedings of a Conference at Leiden University, 25-27 October 2007, Hrsg. G.P.F. Broekman, R.J. Demarée, und O.E. Kaper, 161-202. Leuven: Peeters, 2009. —. "Two Donation Stelae in The Brookly Museum". JARCE 8 (1969-70):59-67.

Koenig, Y. „Notes sur la stèle de donation Caire JE 30972“. ASAE 68 (1982): 111-113.

Malinine, M., G. Posener, und J. Vercoutter. Catalogue des stèles du Sérapéum de Memphis. Paris: Imprimerie Nationale, 1968.

Moje, J. ${ }_{n}$ British Museum EA 73965 und die Sequenz der Lokalregenten des Westdeltas in der 22. Dynastie“. JEgH 4.1 (2011): 81-98.

Montet, P. Le lac sacré de Tanis. Paris: Imprimerie Nationale, 1966.

Quirke, S. und A.J. Spencer. The British Museum Book of Ancient Egypt. London: British Museum Press, 1992.

Ranke, H. Die ägyptischen Personennamen. Band I: Verzeichnis der Namen. Glückstadt: J.J. Augustin, 1935.

Spencer, N. Kom Firin I: The Ramesside Temple and the Site Survey. BM RP 170. London: British Museum, 2008.

Spiegelberg, W. „Neue Schenkungsstelen über Landstiftungen an Tempel“. $z \ddot{A} S{ }_{5}^{6}$ (1920): 55-6o, Taf.4-6.

Winnicki, J. Late Egypt and Her Neighbours. Foreign Population in Egypt in the First Millennium BC. Warschau: Warsaw University, 2009.

Yoyotte, J. „Les principautés du Delta au temps de l'anarchie libyenne“. In Mélanges Maspero I, fasc. 4, 121-181, Taf.1-3. MIFAO 66. Kairo: Institut français d'archéologie orientale du Caire, 1961. 